\title{
State-of-the-art chronic thromboembolic pulmonary hypertension diagnosis and
} management

\author{
D. Jenkins*, E. Mayer ${ }^{\#}$, N. Screaton ${ }^{\top}$ and M. Madani ${ }^{+}$
}

ABSTRACT: Chronic thromboembolic pulmonary hypertension (CTEPH) is a debilitating disease caused by chronic obstruction of pulmonary artery branches following episodes of pulmonary embolism and incomplete thrombus resolution. The prognosis of patients with CTEPH is poor unless an early diagnosis is made and treatment is initiated.

Chest radiography and echocardiography are used in the initial assessment of suspected pulmonary hypertension. A diagnosis of CTEPH may be confirmed by the presence of a mismatched wedge-shaped perfusion deficit during ventilation/perfusion scintigraphy or characteristic findings during multi-slice computed tomography (CT) angiography, including a mosaic perfusion pattern, dilatation of proximal pulmonary arteries and right heart chambers, and the presence of vascular stenosis or obstruction.

Prior to possible surgery, pulmonary angiography remains the definitive diagnostic technique, indicating the site and accessibility of the obstruction. However, many centres utilise CT and magnetic resonance imaging following recent advances in these noninvasive techniques. Haemodynamic evaluation via right heart catheterisation is also mandatory, as pulmonary vascular resistance is the most important determinant of both prognosis and the risk associated with pulmonary endarterectomy surgery.

Accurate CTEPH diagnosis and characterisation of its extent and distribution are imperative to allow the prompt initiation of treatment, particularly surgical pulmonary endarterectomy in eligible patients.

KEYWORDS: Angiography, catheterisation, computed tomography, endarterectomy, pulmonary hypertension, scintigraphy

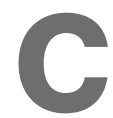
hronic thromboembolic pulmonary hypertension $(\mathrm{CTEPH})$, one of the leading causes of severe pulmonary hypertension (PH), develops from the obstruction of pulmonary artery branches following episodes of pulmonary embolism with incomplete thrombus resolution, formation of fibrosis and remodelling of pulmonary blood vessels [1]. Consequently, pulmonary vascular resistance (PVR) is increased, leading to $\mathrm{PH}$ and progressive right heart failure.

CTEPH is responsible for significant levels of morbidity and mortality, especially when left untreated. Historically, 3-yr mortality has been reported to be as high as $90 \%$ in patients with a mean pulmonary artery pressure $(P \mathrm{pa})$ of $>50 \mathrm{mmHg}$ [2]. Pulmonary endarterectomy (PEA) is the gold standard treatment for CTEPH and represents a potentially curative option in eligible patients [3]. Diagnostic testing is important to distinguish CTEPH from other forms of $\mathrm{PH}$ and to assess operability. Nevertheless, misclassification and delays in diagnosis frequently occur $[4,5]$. In this article we aim to provide an up-to-date review of current diagnostic procedures for CTEPH, using clinical case studies to highlight recommendations and potential pitfalls.

\section{CLINICAL PRESENTATION}

There are no specific signs or symptoms for CTEPH. Patients generally present with progressive dyspnoea on exertion, oedema and/or signs of right heart dysfunction including fatigue, chest pain and syncope $[5,6]$. The clinical presentation of CTEPH does not differ between patients with
AFFILIATIONS

Depts of *Cardiothoracic Surgery, and

"Radiology, Papworth Hospital, Cambridge, UK.

\#Kerckhoff Clinic Heart and Lung Centre, Bad Nauheim, Germany.

+Dept of Cardiothoracic Surgery, University of California San Diego, La Jolla, CA, USA.

CORRESPONDENCE

D. Jenkins

Dept of Cardiothoracic Surgery

Papworth Hospital

Cambridge

CB23 3RE

UK

E-mail: David.Jenkins@

papworth.nhs.uk

Received:

Dec 192011

Accepted after revision:

Jan 062012

PROVENANCE

Publication of this peer-reviewed article was supported by Bayer Pharma AG, Germany (article sponsor, European Respiratory Review issue 123).

Online ISSN 1600-0617 
or without a history of acute pulmonary embolism [6]. While recent registry data from 679 newly diagnosed patients with CTEPH showed a median time from last acute pulmonary embolism to CTEPH diagnosis of 12.5 months [5], the time between the initial acute event and clinical signs of CTEPH can range from a few months to several years.

\section{HISTORY/PHYSICAL EXAMINATION}

CTEPH should be considered in all patients with unexplained $\mathrm{PH}$ and the first step in diagnosis should be obtaining the patient's medical history. Pulmonary embolism is the most common cause of CTEPH. Although it was previously estimated that up to $63 \%$ of patients with CTEPH have no history of acute pulmonary embolism [2], recent data from an international prospective registry of patients with newly diagnosed CTEPH suggest a much lower value. Previous pulmonary embolism was confirmed for $74.8 \%$ of patients and deep-vein thrombosis in 56.1\% [5], which is in line with findings from other recent studies [4]. Other risk factors that may suggest the presence of CTEPH include ventriculo-atrial shunt, indwelling catheters and leads (such as chronic dialysis catheters and pacemakers or automated implantable cardiac defibrillator leads), splenectomy, thyroid replacement therapy, inflammatory bowel disease and a history of malignancy $[7,8]$.

Obtaining the patient's history should be followed by physical and biochemical examination. Subtle physical findings may be evident, such as left parasternal heave, a prominent pulmonary component of S2 or a systolic murmur [9]. Signs of right heart failure, distended neck veins, oedema, ascites and acrocyanosis, may be evident late in the course of CTEPH [9]. In approximately $10 \%$ of patients, subtle bruits over peripheral lung fields are evident [10]. Routine haematological and biochemical tests are usually devoid of abnormalities, although up to $20 \%$ of patients express lupus/anti-cardiolipin antibodies and some may show signs of increased thrombophilic tendency [11, 12]. Normal or slightly reduced pulmonary function may also be evident [13].

As a result of such nonspecific symptoms and often unremarkable physical examination in the early course of CTEPH, diagnostic misclassifications and delays in diagnosis are common $[4,5]$. Symptoms are often mistakenly attributed to deconditioning, advanced age, anxiety or other cardiopulmonary diseases such as obstructive pulmonary disease or ischaemic heart disease. A lack of disease awareness by physicians can also play a role in this delay. Recent registry data indicated that a median time of 14.1 months passed between first symptoms and CTEPH diagnosis [5]. Making the correct diagnosis early is essential, because there is a potential curative treatment in the form of PEA [3]. Indeed, CTEPH is associated with a poor prognosis unless an early diagnosis is made [14].

Case study 1 highlights some of the potential pitfalls faced by physicians in the early diagnosis of CTEPH.

\section{CASE STUDY 1}

A 68-yr-old male complained of exertional breathlessness and presented with World Health Organization class III symptoms. Investigations at his local hospital initially focused on ischaemic heart disease and a coronary angiogram confirmed three-vessel disease. He underwent coronary artery bypass grafting (CABG) at his local cardiac hospital in July 2008, with a complicated post-operative course, but survived after a protracted intensive care unit stay.

Subsequently, he remained very breathless and a ventilation/ perfusion lung scan was performed to exclude a pulmonary embolus. This demonstrated mismatched perfusion defects. A computed tomography (CT) pulmonary angiogram then confirmed evidence of CTEPH and he was referred to his regional $\mathrm{PH}$ unit. A right heart catheter confirmed $\mathrm{PH}$ with a mean $P$ pa of $50 \mathrm{mmHg}$, cardiac output of $5.1 \mathrm{~L} \cdot \mathrm{min}^{-1}$, PVR of $680 \mathrm{dyn} \cdot \mathrm{s} \cdot \mathrm{cm}^{-5}$ and a pulmonary capillary wedge pressure of $12 \mathrm{mmHg}$. A magnetic resonance imaging (MRI) pulmonary artery angiogram confirmed the CT findings, showing a web in the right upper lobe, bilateral lower lobe basal segmental webs and occlusion of the lingular artery.

He was referred to the national centre for PEA surgery and underwent successful PEA in April 2011. Post-operatively his $P$ pa decreased to a mean of $24 \mathrm{mmHg}$, with a cardiac output of $7 \mathrm{~L} \cdot \mathrm{min}^{-1}$ and PVR of $160 \mathrm{dyn} \cdot \mathrm{min}^{-1} \cdot \mathrm{cm}^{-5}$. He made an uneventful recovery and was in WHO class I at his 3-month post-operative reassessment.

\section{Key learning points}

The learning point from this case is that the original history was misinterpreted and when investigations demonstrated ischaemic heart disease, $\mathrm{PH}$ was not considered. The patient was fortunate to survive the CABG procedure and this operation made his definitive treatment more complex as he required redo surgery.

\section{IMAGING AND HAEMODYNAMIC EVALUATION}

Following physical and biochemical assessment of patients with suspected $\mathrm{PH}$, confirmation of CTEPH diagnosis usually requires radiological investigations. In patients being investigated for unexpected cardiorespiratory symptoms, the diagnosis of PH may first be suggested on the basis of imaging, particularly in the form of CT.

\section{Chest radiography}

Patients with suspected $\mathrm{PH}$ usually undergo chest radiography and echocardiography. Although many patients show relatively normal findings on chest radiographs, signs of $\mathrm{PH}$ include enlargement of the central pulmonary arteries or right atrium, and signs suggestive of thromboembolic aetiology include attenuation and amputation of the lobar or segmental pulmonary arteries [15].

\section{Echocardiography}

Echocardiography is widely used as the initial diagnostic tool for PH. Although it is not specific for the diagnosis of CTEPH, it enables indirect assessment of $P$ pa and permits exclusion of intracardiac shunt or left heart disease as a cause for $\mathrm{PH}$. Indications of $\mathrm{PH}$ may include: right ventricular dilatation, hypertrophy and hypokinesis, right atrial enlargement, right ventricular pressure overload, and tricuspid regurgitation. Lack of signs of right ventricular overload observed by echocardiography in conjunction with a normal N-terminal pro-brain natriuretic protein level virtually excludes significant PH [16]. The European Society of Cardiology (ESC) and 
European Respiratory Society (ERS) guidelines for the diagnosis and treatment of $\mathrm{PH}$ recommend a follow-up echocardiography in patients with acute pulmonary embolism showing signs of $\mathrm{PH}$ or right ventricular dysfunction to determine whether or not $\mathrm{PH}$ has resolved [17].

\section{Ventilation/perfusion lung scan}

A normal ventilation/perfusion scan essentially rules out a diagnosis of CTEPH. The absence of perfusion to one lung increases the suspicion of other disorders such as malignancy, mediastinal fibrosis or vasculitis. In the setting of $\mathrm{PH}$, a normal ventilation scan with a wedge-shaped perfusion deficit is characteristic of CTEPH (fig. 1) [18]. Recent data from a CTEPH registry showed that $98.7 \%$ of patients had abnormal perfusion scans and $19.0 \%$ had abnormal ventilation scans [5]. Such findings should be followed by further diagnostic studies since ventilation/perfusion scanning might underestimate the burden of vascular obstruction [9]. Furthermore, although ventilation/perfusion scanning is a functional technique, it has limited spatial resolution [19].

\section{Computed tomography}

CT has the advantage of being a noninvasive, cross-sectional technique with a spatial resolution close to conventional pulmonary angiography. In patients with CTEPH, CT pulmonary angiography may reveal organised thrombi lining the proximal pulmonary vessels, abrupt tapering or amputation of vessels or subtle intraluminal fibrous webs (fig. 2). Enlarged bronchial artery collaterals (not usually found with other types of $\mathrm{PH}$ ) may be seen in CTEPH and are considered a good prognostic sign in operable patients [18, 20-22]. Other findings a)

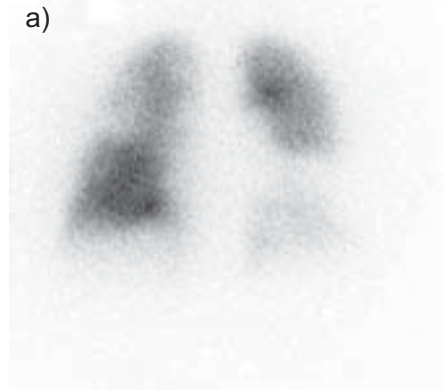

c)

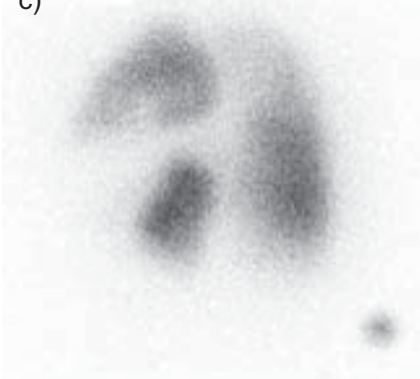

b)

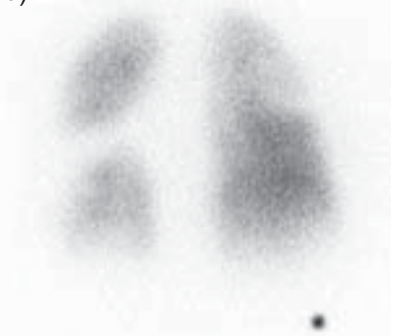

d)
FIGURE 1. Perfusion scintigraphy of a patient with chronic thromboembolic pulmonary hypertension showing multiple wedge-shaped perfusion defects. The ventilation study (not shown) was normal. a) Anterior, b) posterior, c) left posterior oblique and d) right posterior oblique. include dilatation of proximal pulmonary arteries and right heart chambers, scarring and a mosaic perfusion pattern [5].

Modern multi-row CT scanners are widely available and permit rapid acquisition of a thin-slice dataset even in breathless patients. CT pulmonary angiography is now considered the reference standard in acute pulmonary embolism and can accurately define the nature and extent of disease in CTEPH, and provide multi-planar and three-dimensional reconstructions of the vascular tree. The recent development of dual-energy CT permits the identification of perfusion defects distal to proximal vascular obstruction and, therefore, has the potential to improve the detection of distal CTEPH [23]. Web disease is also clearly observed with CT pulmonary angiography. This technique may have a role in the future in helping to identify those patients suitable for PEA.

However, outside experienced centres, CT angiography can be associated with difficulties regarding the diagnosis and evaluation of operability in CTEPH. A systematic approach to interpretation is required by a radiologist familiar with the condition [21] and like ventilation/perfusion scanning, CT angiography might underestimate the clot burden [9]. Multidetector CT angiography has also been shown to be less sensitive than ventilation/perfusion scintigraphy at detecting CTEPH [19].

Once ventilation/perfusion scanning and/or CT angiogram show signs compatible with CTEPH, the patient should be referred to a centre with expertise in the medical and surgical management of this condition. Centres with a high volume of patients tend to obtain the best outcomes and can offer advanced therapy such as PEA, as well as enrolment in clinical trials of medical therapies for those patients who are ineligible for surgery, a strategy recommended in the ESC/ERS guidelines [17].

The potential for CT angiography to underestimate the clot burden in patients with CTEPH is highlighted in case study 2.

\section{CASE STUDY 2}

A 46-yr-old male was referred to a specialist centre for further evaluation. At 25 yrs of age he had suffered cardiac arrest during a knee procedure and was found to have bradycardia and sick sinus syndrome. He was fitted with a pacemaker that subsequently became infected within the first 6 months. Although a second pacemaker was fitted, he continued to have progressively worsening dyspnoea on exertion. Echocardiography continued to show worsening $\mathrm{PH}$ and right ventricular function, resulting in the placement of a bi-ventricular pacer. Over the following 20 yrs he was documented as having some segmental and subsegmental pulmonary emboli.

Prior to referral, the patient was treated with multiple medical regimens, including sildenafil. With no relief of symptoms, he ultimately underwent a ventilation/perfusion scan, which confirmed evidence of significant thromboembolic disease. Anticoagulation therapy was administered and he underwent right heart catheterisation. CT angiography did not indicate any significant thromboembolic disease, although some segmental and subsegmental intraluminal defects were apparent.

Upon referral, he received repeat right heart catheterisation that showed a mean $P$ pa of $34 \mathrm{mmHg}$, PVR of $344 \mathrm{dyn} \cdot \mathrm{s} \cdot \mathrm{cm}^{-5}$, right atrial pressure of $13 \mathrm{mmHg}$ and cardiac output of 

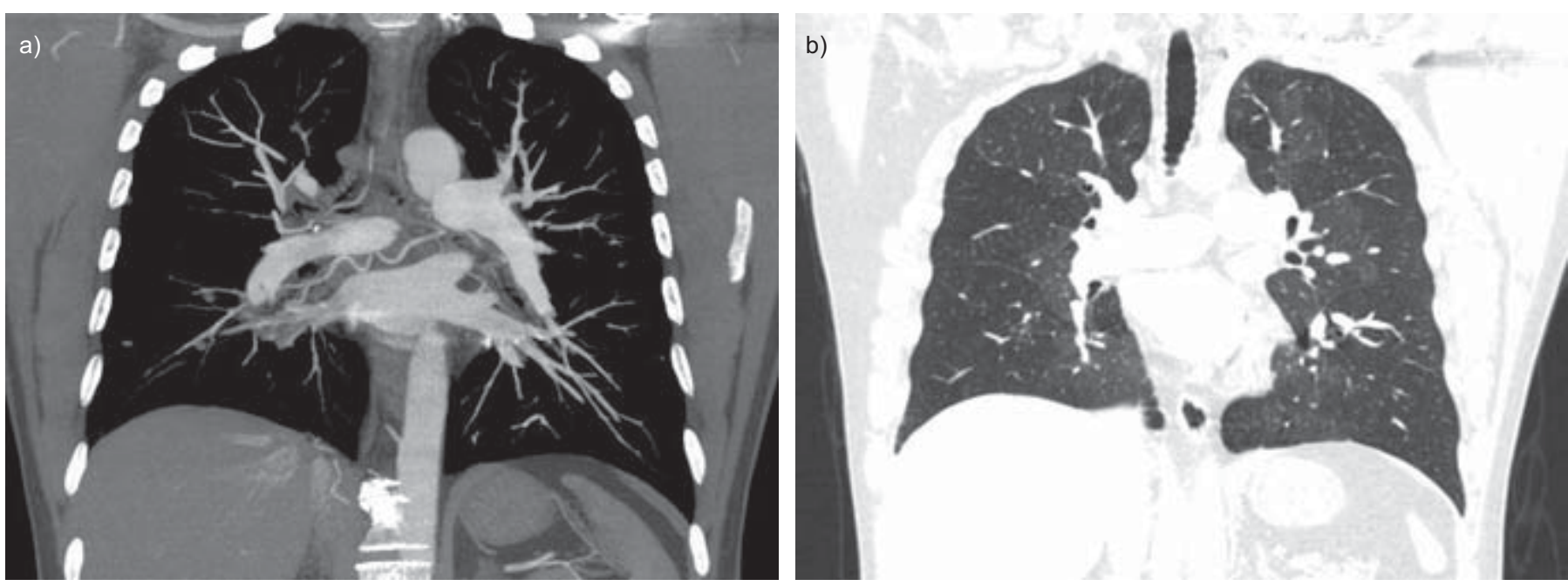

FIGURE 2. a) Maximal intensity projection from a computed tomography pulmonary arteriogram showing amputation of the basal segment of the right lower lobe and web disease in the left lower lobe in keeping with proximal chronic thromboembolic pulmonary hypertension (CTEPH). Enlargement of the bronchial arteries is also visible. b) Wedge-shaped areas of reduced attenuation in the lung parenchyma indicative of mosaic perfusion consistent with CTEPH.

$5.1 \mathrm{~L} \cdot \mathrm{min}^{-1}$. Based on a pulmonary angiogram, PEA was offered with a minimal risk. The patient subsequently underwent uneventful PEA, all his existing leads were removed and tricuspid repair and epicardial leads were introduced. He was discharged 10 days later and his post-operative course was completely unremarkable. There was a considerable improvement in post-operative haemodynamics: mean $P$ pa was $8 \mathrm{mmHg}$, PVR was $152 \mathrm{dyn} \cdot \mathrm{s} \cdot \mathrm{cm}^{-5}$, right atrial pressure was $8 \mathrm{mmHg}$ and cardiac output was $5.8 \mathrm{~L} \cdot \mathrm{min}^{-1}$. Dramatic improvements were also evident on follow-up ventilation/ perfusion scans and echocardiography, and sildenafil therapy was discontinued.

\section{Key learning points}

This case highlights an example of where the degree of thromboembolic disease shown on both CT and pulmonary angiography did not correlate with the extent of disease found during surgery. Surgery should always be considered when there is evidence of thromboembolic disease. Furthermore, potential candidates should be referred to an experienced surgical centre where, if appropriate, surgery should be offered without delay.

\section{Right heart catheterisation/haemodynamic evaluation}

In addition to aiding the procedure of pulmonary angiography, right heart catheterisation provides accurate prognostic information. Furthermore, it can provide additional information on disease severity, right heart function and mixed venous oxygen saturation [24]. Haemodynamic evaluation via right heart catheterisation is mandatory in diagnosing CTEPH.

A mean $P$ pa $\geqslant 25 \mathrm{mmHg}$, a pulmonary capillary wedge pressure $\leqslant 15 \mathrm{mmHg}$ and PVR $>2$ Wood units confirms diagnosis of CTEPH in patients with chronic or organised thromboembolic obstructions [17]. It is essential to relate haemodynamic values from right heart catheterisation to vascular obstructions seen by imaging. When assessing the operability of CTEPH, PVR that is excessively elevated compared with the level of visible obstruction is generally indicative of increased risk and in some cases inoperable smallvessel disease. It has been reported that analysis of the decay curve of the wedge tracing following balloon occlusion at right heart catheterisation may help distinguish more distal inoperable small vessel disease from more proximal operable segmental disease by partitioning the PVR [25]. However, this technique may not be reproducible in all patients and, unfortunately, has not been found to be a useful discriminator in routine clinical practice.

PVR at both diagnosis and discharge from intensive care correlates with in-hospital and 1-yr mortality following PEA [26]. In-hospital and 1-yr mortality rates can be as high as $11 \%$ and $13 \%$, respectively, for those patients with a PVR $>1,200 \mathrm{dyn} \cdot \mathrm{s} \cdot \mathrm{cm}^{-5}$. Post-operative residual $\mathrm{PH}$ has been identified as the most important predictor of death, with a mortality rate of $31 \%$ witnessed in patients with a postoperative PVR $>500 \mathrm{dyn} \cdot \mathrm{s} \cdot \mathrm{cm}^{-5}$ compared with $1 \%$ in those with a post-operative PVR $<500 \mathrm{dyn} \cdot \mathrm{s} \cdot \mathrm{cm}^{-5}$ [3].

\section{Pulmonary angiography}

Pulmonary angiography is still considered to be the gold standard diagnostic procedure for defining the extent and distribution of disease in CTEPH and it has a relatively good safety profile, even in patients with severe PH. Findings typically include dilatation of the pulmonary artery, vascular obstructions, vascular webs, post-obstructive dilatations and poorly perfused areas of the lung (fig. 3) [18]. Proximal lesions of the pulmonary artery are evident in $\sim 63 \%$ of patients [5]. Pulmonary angiography is often performed in conjunction with right heart catheterisation [9]. If right heart catheterisation is carried out first, the same introducer sheath can be used for pulmonary angiography, thereby reducing risk and inconvenience for the patient. It is recommended that both right heart catheterisation and pulmonary angiography should be carried out by experienced staff at the same institution as the surgery is to take place or at a unit with specialist experience with investigation of patients with $\mathrm{PH}$ (fig. 3) [9]. 

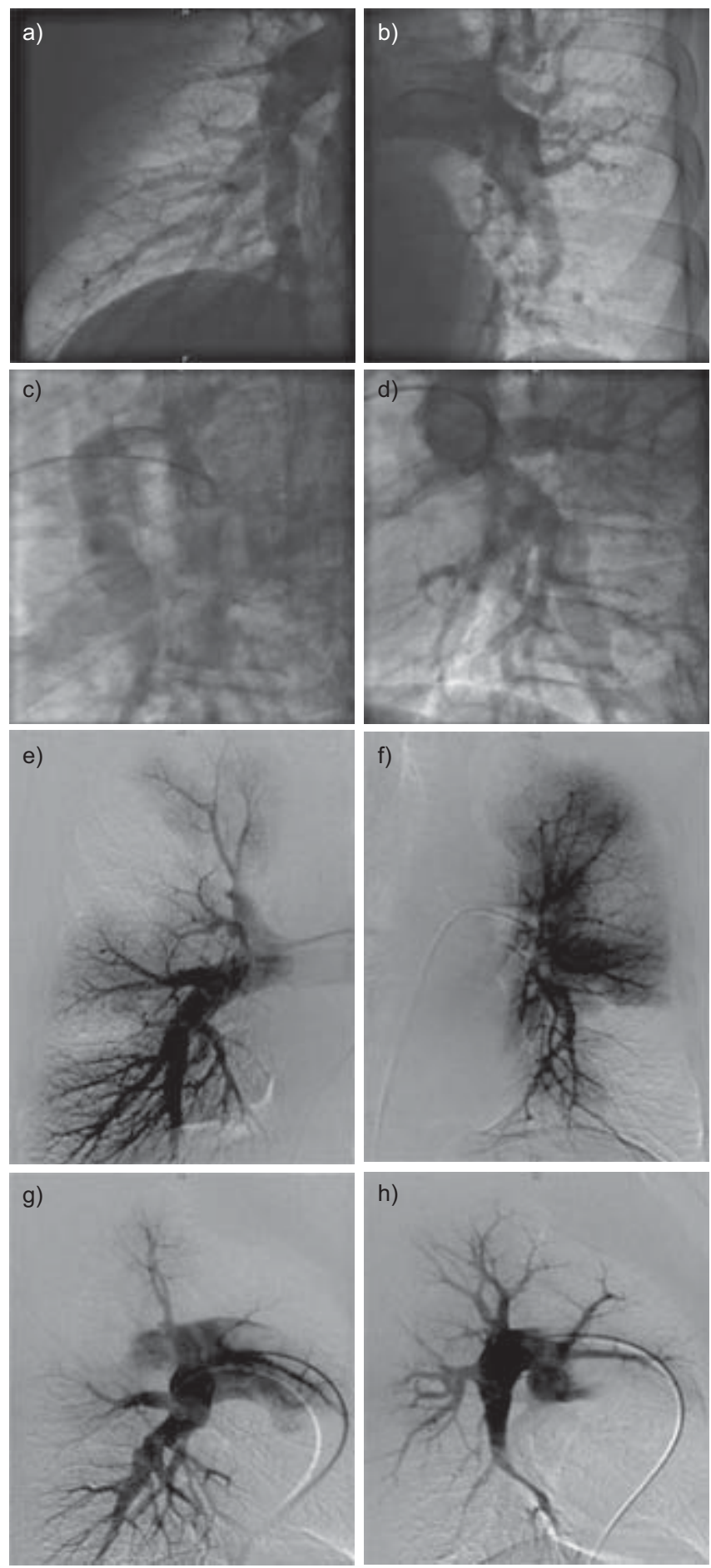

FIGURE 3. Pulmonary angiography in the right lung (a, c, e and g) and the left lung ( $b, d, f$ and $h$ ) of a patient with chronic thromboembolic pulmonary hypertension. The first image $(a-d)$ was taken at a non-specialist centre with suboptimal vascular opacificaiton and the patient was considered inoperable. The second pulmonary angiography (e-h) was undertaken at a specialist surgical centre with selective angiography showing proximal disease, and resulted in the patient receiving successful pulmonary endarterectomy surgery. a, b, e, f) Anterior view and c, d, g, h) lateral views.

Recent advances in cross-sectional techniques such as CT and MRI can provide details not only of the pulmonary artery lumen, but also the vascular wall, structure of the pulmonary parenchyma and heart. MRI can also provide information on right and left ventricular function and estimate cardiac output. Such advances have led to some centres adopting these techniques as primary investigations with conventional pulmonary angiography acting as a definitive problem-solving technique in cases of borderline operability or where investigation such as MRI is contraindicated. However, pulmonary angiography remains the definitive diagnostic technique for the work-up of CTEPH prior to surgery, indicating the site and accessibility of the obstruction (fig. 3). Pulmonary angiography should be performed in all patients who have an abnormal ventilation/perfusion scan and in those in whom imaging findings on CT and/or MRI are equivocal. In such patients, surgery should not be ruled out without a complete and comprehensive imaging evaluation that includes at least two imaging modalities.

The importance of a comprehensive imaging evaluation by specialist radiologists when determining operability is underlined in case study 3.

\section{CASE STUDY 3}

A 58-yr-old male with a history of deep-vein thrombosis, acute pulmonary embolism and increasing dyspnoea presented with suspicion of CTEPH in February 2011. Initial diagnosis took place at his local hospital in August 2011. Echocardiography revealed enlarged right heart chambers and a right ventricular systolic pressure of $80 \mathrm{mmHg}$. Peripheral perfusion deficits were evident following CT pulmonary angiography, coupled with no central embolus in the main arteries. PVR, measured by right heart catheterisation, was $768 \mathrm{dyn} \cdot \mathrm{s} \cdot \mathrm{cm}^{-5}$. Following pulmonary angiography (fig. 3), he was diagnosed with distal CTEPH and considered to be inoperable.

A second opinion was sought at a PEA reference centre, where the patient underwent a second pulmonary angiography (fig. 3) and was considered suitable for surgery. In October 2011, he underwent successful PEA. His Ppa decreased to a mean of $36 \mathrm{mmHg}$ following surgery from a pre-operative value of $46 \mathrm{mmHg}$, and his PVR decreased from 412 dyn $\cdot \mathrm{s} \cdot \mathrm{cm}^{-5}$ to $266 \mathrm{dyn} \cdot \mathrm{s} \cdot \mathrm{cm}^{-5}$. He had an uneventful postoperative course. Mean $P$ pa continued to decrease, eventually reaching $27 \mathrm{mmHg}$. The patient was discharged on postoperative day 14 .

\section{Key learning points}

There are two key learning points from this case. First, a second opinion is essential in patients who are initially considered inoperable. Secondly, such patients should always be evaluated at an expert surgical centre where top-level diagnosis by specialist radiologists and physicians is available.

\section{Magnetic resonance angiography}

Although MRI has limited availability and is relatively expensive and time-consuming, it is a noninvasive technique with no radiation exposure and offers great potential in CTEPH diagnosis (fig. 4). It can be used for morphological, anatomical and functional assessment of both the heart and pulmonary circulation. Both high-resolution pulmonary angiography and dynamic temporally resolved angiography can be performed, with the latter enabling the detection of perfusion defects [27]. Furthermore, since it does not require 

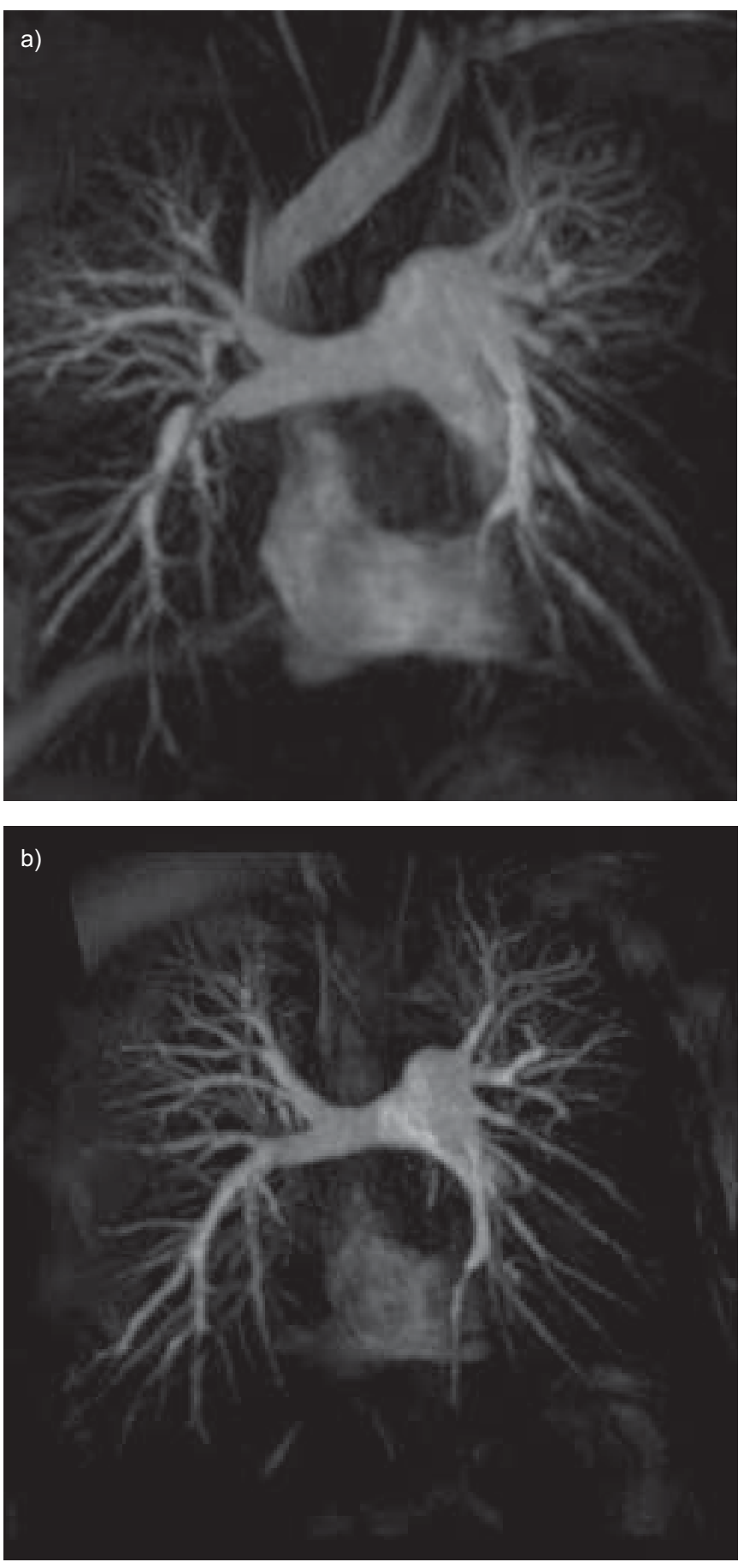

FIGURE 4. Magnetic resonance angiography from a patient with chronic thromboembolic pulmonary hypertension a) pre- and b) post-endarterectomy. Unlike other forms of imaging, magnetic resonance angiography does not utilise ionising radiation and is useful for follow-up examinations.

exposure to radiation and nephrotoxic contrast agents it is suitable for repeated studies (fig. 4) [21]. Thus, the use of this technique for assessment of patients with CTEPH is increasing.

\section{Others}

Pulmonary angioscopy is a specialist technique that is not routinely used in CTEPH diagnosis, but can help to resolve the differential diagnosis between primary $\mathrm{PH}$ and distal/small-vessel pulmonary thromboembolic disease [15]. However, with recent advances in CT imaging and pulmonary angiography, pulmonary angioscopy is rarely used in the diagnosis of CTEPH.

Invasive coronary angiography, pulmonary function tests and cardiopulmonary exercise testing are occasionally useful to evaluate certain patients with established CTEPH or to rule out alternative or concomitant diagnoses. Coronary angiography is also important in patients with risk factors for ischaemic heart disease prior to PEA as concomitant coronary artery bypass grafts may be necessary at the time of PEA. Exercise testing, in the form of the 6-min walk test, is often utilised to assess prognosis and response to therapy. It has been shown to accurately reflect the clinical and haemodynamic severity of the disease [28].

\section{CTEPH TREATMENT}

CTEPH is an obstructive disease and PEA is the only approved and potentially curative treatment currently available. Therefore, surgical evaluation and cure by means of PEA at an experienced centre should be offered to all patients who have $\mathrm{PH}$ with evidence of thromboembolic disease. However, surgery may not be offered to up to $40 \%$ of patients due to distal or inaccessible thromboembolism or comorbidities [26, 29], particularly at inexperienced centres. The experience of the PEA team determines which lesions are considered as surgically treatable and accessibility is dependent on the skills and experience of the surgeon, in addition to the angiographic appearance. Furthermore, the degree of $\mathrm{PH}$ and its correlation to the degree of thromboembolic obstruction, or "clot burden", is an important factor in determining eligibility and prognosis.

Anticoagulation is prescribed in all patients with CTEPH to prevent in situ pulmonary artery thrombosis and recurrent venous thromboembolism. Medical pre-treatment with specific $\mathrm{PAH}$ therapies is also used as a bridge to PEA, even though evidence of the efficacy of such therapies from randomised clinical trials in patients with CTEPH is lacking [29]. Indeed, at the time of CTEPH diagnosis, $37.9 \%$ of patients in an international registry were receiving at least one pulmonary arterial hypertension-targeted therapy [5]. Unfortunately, this practice may result in a delay of referral for surgery and unnecessarily increase the risk of such an operation. There is currently no evidence from randomised clinical trials to support medical treatment of CTEPH as an alternative to PEA, or as a bridge to PEA, as the only trial performed failed to show efficacy in the primary end-point [30]. Further studies are necessary to obtain reliable long-term data on the effects of medical therapies in patients with CTEPH; therefore, only patients who are considered inoperable by experienced centres should be enrolled in randomised controlled clinical trials [17]. Only two agents are currently under investigation in inoperable CTEPH (see www.clinicaltrials.gov). There are also anecdotal reports of a more difficult endarterectomy, due to clot changes following pre-treatment; although this remains to be confirmed in large clinical studies.

Case study 4 illustrates the dangers of delaying surgery in patients with operable CTEPH.

\section{CASE STUDY 4}

A 47-yr-old male with a history of hypertension and progressive dyspnoea (which did not improve with weight loss) 
was found to have $\mathrm{PH}$ and right ventricular dysfunction on routine echocardiography. Further investigation led to a diagnosis of CTEPH. Anticoagulant therapy (warfarin) and bosentan were prescribed and he was subsequently referred to a specialist centre for surgery.

Initial right heart catheterisation showed a mean $P$ pa of $57 \mathrm{mmHg}$, PVR of $635 \mathrm{dyn} \cdot \mathrm{s} \cdot \mathrm{cm}^{-5}$, right atrial pressure of $4 \mathrm{mmHg}$, pulmonary capillary wedge pressure of $12 \mathrm{mmHg}$, cardiac output of $5.6 \mathrm{~L} \cdot \mathrm{min}^{-1}$ and mixed venous oxygen saturation of $68 \%$. Pulmonary angiography revealed significant CTEPH, which was deemed to be operable. He was offered surgery with a very low risk and a high likelihood of cure; however, following consultation with his local physician, he elected to delay surgery. His local physician recommended continuation of medical therapy and the patient complied and returned home.

Over the course of the next 20 months, the patient's condition deteriorated significantly. He now displayed class IV symptoms and was referred back to the specialist centre for reevaluation. He underwent repeat right heart catheterisation, which revealed considerable deterioration of haemodynamic parameters: mean P pa $69 \mathrm{mmHg}$, PVR 1,658 dyn $\cdot \mathrm{s} \cdot \mathrm{cm}^{-5}$, right atrial pressure $11 \mathrm{mmHg}$, pulmonary capillary wedge pressure $12 \mathrm{mmHg}$, cardiac output $2.7 \mathrm{~L} \cdot \mathrm{min}^{-1}$ and mixed venous oxygen saturation $56 \%$. Repeat pulmonary angiography revealed no evidence of new thromboembolic disease but indicated that small-vessel vasculopathy had developed. Surprisingly, despite medical management, there was significant deterioration of the patient's condition and worsening $\mathrm{PH}$ and right ventricular function. The offer of surgery, this time with a higher risk, was accepted.

Surgery was successful and the patient was discharged 15 days later. Post-operative haemodynamic parameters showed significant improvements: mean $P$ pa was $29 \mathrm{mmHg}$, PVR was $330 \mathrm{dyn} \cdot \mathrm{s} \cdot \mathrm{cm}^{-5}$, right atrial pressure was $8 \mathrm{mmHg}$ and cardiac output was $4.6 \mathrm{~L} \cdot \mathrm{min}^{-1}$. Improvements were also evident on ventilation/perfusion lung scans and echocardiography, resulting in the discontinuation of bosentan therapy. The patient continued to do well during follow-up.

\section{Key learning points}

Although this patient did well with surgery, his risks were much higher at the second presentation, and the outcome would have been better had he accepted surgery at the initial presentation. Clearly there was progression of $\mathrm{PH}$ and right ventricular failure, despite no evidence of new thromboembolic disease and despite medical management. Candidates should be referred to an experienced centre for surgery once the diagnosis of CTEPH has been confirmed. Furthermore, if appropriate, patients should be offered surgery without delay.

\section{CONCLUSIONS}

Although CTEPH is one of the leading causes of severe $\mathrm{PH}$, it remains underdiagnosed. These delays in diagnosis contribute to the poor prognosis associated with the disease.

Diagnosis of CTEPH requires input from several radiological techniques. Echocardiography, ventilation/perfusion scintigraphy and CT angiography are all essential in the initial diagnosis of CTEPH. Pulmonary angiography remains the gold standard diagnostic technique for assessing operability but recent advances in CT and MRI angiography show great promise. Furthermore, haemodynamic evaluation by right heart catheterisation provides vital prognostic information and an estimate of the relative risk of PEA surgery.

CTEPH should be considered in all patients with $\mathrm{PH}$ as early diagnosis helps to identify those patients suitable for PEA, a potentially curative treatment. All patients with suspected CTEPH should be referred to an expert centre for a proper diagnostic evaluation to exclude or confirm the diagnosis and assess operability.

\section{STATEMENT OF INTEREST}

E. Mayer has received fees for speaking and consultancy from Actelion and Bayer Pharma.

\section{ACKNOWLEDGEMENTS}

The authors received editorial assistance from Adelphi Communications (Bollington, UK), funded by Bayer Pharma AG (Berlin, Germany).

\section{REFERENCES}

1 Moser KM, Auger WR, Fedullo PF. Chronic major-vessel thromboembolic pulmonary hypertension. Circulation 1990; 81: 1735-1743.

2 Dalen JE, Alpert JS. Natural history of pulmonary embolism. Prog Cardiovasc Dis 1975; 17: 259-270.

3 Jamieson SW, Kapelanski DP, Sakakibara N, et al. Pulmonary endarterectomy: experience and lessons learned in 1,500 cases. Ann Thorac Surg 2003; 76: 1457-1462.

4 D'Armini AM, Cattadori B, Monterosso C, et al. Pulmonary thromboendarterectomy in patients with chronic thromboembolic pulmonary hypertension: hemodynamic characteristics and changes. Eur J Cardiothorac Surg 2000; 18: 696-701.

5 Pepke-Zaba J, Delcroix M, Lang I, et al. Chronic thromboembolic pulmonary hypertension (CTEPH): results from an international prospective registry. Circulation 2011; 124: 1973-1981.

6 Rubin LJ, Badesch DB. Evaluation and management of the patient with pulmonary arterial hypertension. Ann Intern Med 2005; 143: 282-292.

7 Bonderman D, Jakowitsch J, Adlbrecht C, et al. Medical conditions increasing the risk of chronic thromboembolic pulmonary hypertension. Thromb Haemost 2005; 93: 512-516.

8 Bonderman D, Wilkens H, Wakounig S, et al. Risk factors for chronic thromboembolic pulmonary hypertension. Eur Respir J 2009; 33: 325-331.

9 Hoeper MM, Mayer E, Simonneau G, et al. Chronic thromboembolic pulmonary hypertension. Circulation 2006; 113: 2011-2020.

10 ZuWallack RL, Liss JP, Lahiri B. Acquired continuous murmur associated with acute pulmonary thromboembolism. Chest 1976; 70: 557-559.

11 Wolf M, Boyer-Neumann C, Parent F, et al. Thrombotic risk factors in pulmonary hypertension. Eur Respir J 2000; 15: 395-399.

12 Lang I, Kerr K. Risk factors for chronic thromboembolic pulmonary hypertension. Proc Am Thorac Soc 2006; 3: 568-570.

13 Suntharalingam J, Machado RD, Sharples LD, et al. Demographic features, BMPR2 status and outcomes in distal chronic thromboembolic pulmonary hypertension. Thorax 2007; 62: 617-622.

14 Riedel M, Stanek V, Widimsky J, et al. Longterm follow-up of patients with pulmonary thromboembolism - late prognosis and evolution of hemodynamic and respiratory data. Chest 1982; 81: 151-158.

15 Madani MM, Jamieson SW. Surgical treatment of chronic thromboembolic pulmonary hypertension. Adv Pulmon Hyperten 2007; 6: 83-91. 
16 Klok FA, Huisman MV. Epidemiology and management of chronic thromboembolic pulmonary hypertension. Neth $J$ Med 2010; 68: 347-351.

17 Galiè N, Hoeper MM, Humbert M, et al. Guidelines for the diagnosis and treatment of pulmonary hypertension. Eur Respir J 2009; 34: 1219-1263.

18 Coulden R. State-of-the-art imaging techniques in chronic thromboembolic pulmonary hypertension. Proc Am Thorac Soc 2006; 3: 577-583.

19 Tunariu N, Gibbs SJ, Win Z, et al. Ventilation-perfusion scintigraphy is more sensitive than multidetector CTPA in detecting chronic thromboembolic pulmonary disease as a treatable cause of pulmonary hypertension. J Nucl Med 2007; 48: 680-684.

20 Kauczor HU, Schwickert HC, Mayer E, et al. Spiral CT of bronchial arteries in chronic thromboembolism. J Comput Assist Tomogr 1994; 18: 855-861

21 McNeil K, Dunning J. Chronic thromboembolic pulmonary hypertension (CTEPH). Heart 2007; 93: 1152-1158.

22 Ley S, Kreitner KF, Morgenstern I, et al. Bronchopulmonary shunts in patients with chronic thromboembolic pulmonary hypertension: evaluation with helical CT and MR imaging. AJR Am J Roentgenol 2002; 179: 1209-1215.

23 Hoey ET, Mirsadraee S, Pepke-Zaba J, et al. Dual-energy CT angiography for assessment of regional pulmonary perfusion in patients with chronic thromboembolic pulmonary hypertension: initial experience. AJR Am J Roentgenol 2011; 196: 524-532.
24 Hoeper MM, Barberà JA, Channick RN, et al. Diagnosis, assessment, and treatment of non-pulmonary arterial hypertension pulmonary hypertension. J Am Coll Cardiol 2009; 54: Suppl. 1, S85-S96.

25 Kim NH, Fesler P, Channick RN, et al. Preoperative partitioning of pulmonary vascular resistance correlates with early outcome after thromboendarterectomy for chronic thromboembolic pulmonary hypertension. Circulation 2004; 109: 18-22.

26 Mayer E, Jenkins D, Lindner J, et al. Surgical management and outcome of patients with chronic thromboembolic pulmonary hypertension: results from an international prospective registry. J Thorac Cardiovasc Surg 2011; 141: 702-710.

27 Kreitner KF, Ley S, Kauczor HU, et al. Chronic thromboembolic pulmonary hypertension: pre- and postoperative assessment with breath-hold MR imaging techniques. Radiology 2004; 232: 535-543.

28 Reesink HJ, van der Plas MN, Verhey NE, et al. Six-minute walk distance as parameter of functional outcome after pulmonary endarterectomy for chronic thromboembolic pulmonary hypertension. J Thorac Cardiovasc Surg 2007; 133: 510-516.

29 Mayer E. Surgical and post-operative treatment of chronic thromboembolic pulmonary hypertension. Eur Respir Rev 2010; 19: 64-67.

30 Jaïs X, D'Armini AM, Jansa P, et al. Bosentan for treatment of inoperable chronic thromboembolic pulmonary hypertension: BENEFiT (Bosentan Effects in iNopErable Forms of chronIc Thromboembolic pulmonary hypertension), a randomized, placebo-controlled trial. J Am Coll Cardiol 2008; 52: 2127-2134. 